\title{
Aggregation of Transmembrane Peptides Studied by Spin-Label EPR
}

\author{
Francesco Scarpelli, ${ }^{\dagger}$ Malte Drescher, ${ }^{\dagger, \perp}$ Tania Rutters-Meijneke, ${ }^{*}$ Andrea Holt, \\ Dirk T. S. Rijkers, ${ }^{8}$ J. Antoinette Killian, ${ }^{\ddagger}$ and Martina Huber*, \\ Department of Molecular Physics, Huygens Laboratory, Leiden University, P.O. Box 9504, 2300 RA Leiden, \\ The Netherlands, Chemical Biology \& Organic Chemistry, Bijvoet Center for Biomolecular Research, Utrecht \\ University, Utrecht, The Netherlands, and Medicinal Chemistry and Chemical Biology, Utrecht Institute for \\ Pharmaceutical Sciences, Department of Pharmaceutical Sciences, Faculty of Science, Utrecht University, \\ Utrecht, The Netherlands
}

\begin{abstract}
The structure and function of membrane proteins is partly determined by the interaction of these proteins with the lipids of the membrane. Peptides forming single membrane-spanning $\alpha$-helices, such as the WALP peptide (acetyl-GWWL(AL) ${ }_{n}$ WWA-amide), are good models for such interactions. This interaction can be studied by investigating the aggregation of peptides. If the peptides remain isolated in the membrane, the peptide-lipid interaction dominates, if the peptides aggregate, the peptide-peptide interaction is stronger. The intrinsic dynamics and the disordered nature of the system require new approaches to determine eventual aggregation. We performed electron paramagnetic resonance (EPR) on spin-labeled WALP (SL-WALP) in the gel and the liquid-crystalline phases of two different phospholipids, the saturated DPPC (1,2-dipalmitoyl$s n$-glycero-3-phosphocholine), and the unsaturated DOPC (1,2-dioleoyl-sn-glycero-3-phosphocholine). At low temperatures $(120 \mathrm{~K})$ where both lipids are in the gel phase, less extensive aggregation is observed for the peptide in DOPC as compared to DPPC. Together with previous data on aggregation of WALP peptides from atomic force microscopy and fluorescence spectroscopy at $294 \mathrm{~K}$ (Sparr; et al. Biochemistry 2005, 44, $2-10$ ), the results suggest that at $120 \mathrm{~K}$ WALP peptides form line aggregates in DOPC and cluster aggregates in DPPC. In the liquid-crystalline phase of both lipids, signatures of aggregation are absent, showing that in this phase the peptide can be accommodated by either lipid. It can be concluded that the lipid phase, in this case gel or liquid-crystalline, is a more important determinant for peptide aggregation than whether the lipid is saturated (DPPC) or unsaturated (DOPC). In view of the gel-phase-like behavior of some membrane phases in physiological systems the methodology should be relevant.
\end{abstract}

\section{Introduction}

The assembly and folding of membrane proteins, and the association of these proteins inside the membrane depend strongly on the balance between protein - protein and protein-lipid interactions. Peptides forming transmembrane (TM) $\alpha$-helices and their interactions with phospholipid bilayers serve as models to define the elementary aspects of the protein-membrane interactions governing the properties of TM proteins (Figure 1a). The WALP peptide (acetyl-GWWL(AL) ${ }_{n}$ WWA-amide) is one of the paradigmatic TM peptides and its properties have been studied in various membrane environments. ${ }^{1-6}$

The response of peptides and ultimately membrane proteins to changes in membrane conditions depends on the balance of peptide-peptide, peptide-lipid, and lipid-lipid interactions. Consequently, the type of lipid (saturated vs unsaturated) and the membrane phase (gel phase vs liquid-crystalline phase) are important factors. One of the intriguing and potentially biologically relevant responses of the peptides to different membrane conditions is the lateral association of TM peptides within the membrane (Figure 1b,c), which we will, in the following, refer to as aggregation. ${ }^{2,7}$ Since membrane systems are intrinsically

* To whom correspondence should be addressed. Phone: +31 71527 5560. Fax: +31 715275819. E-mail: mhuber@molphys.leidenuniv.nl.

${ }^{\dagger}$ Leiden University.

* Bijvoet Center for Biomolecular Research, Utrecht University.

${ }^{\S}$ Utrecht Institute for Pharmaceutical Sciences, Utrecht University.

Department of Chemistry, University of Konstanz, Germany. a)

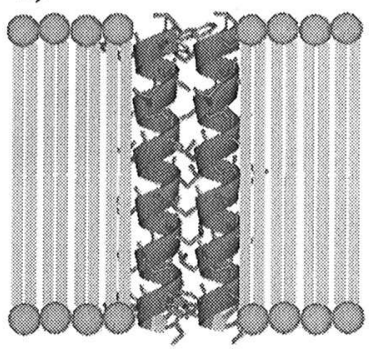

b)

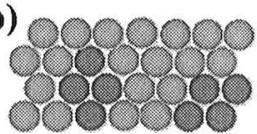

c)

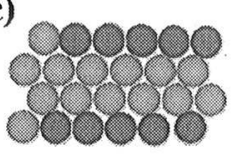

Figure 1. Schematic representation of WALP peptides in a gel-phase lipid bilayer. (a) Lipid bilayer, lipid head groups (gray), lipid chains (yellow), WALP helices (red), side view. (b) Peptide cluster aggregates, top view. (c) Peptide linear aggregates, top view. AFM ${ }^{1,10}$ shows that the spacing between lines is $8 \mathrm{~nm}$; thus separations between lines are not to scale.

disordered and dynamic, many conventional methods for structure investigation cannot be employed to monitor peptide aggregation. Also, often these methods can only probe aggregation in certain lipids or phases of the membrane, for example, because the phase of interest occurs in an inaccessible temperature range. Thus, a combination of methods is needed to address the question of how the balance of forces in peptidemembrane interactions can influence peptide aggregation.

For WALP peptides, it has been shown by atomic force microscopy (AFM) and fluorescence techniques that they form 
TABLE 1: Peptides and Their Amino Acidic Sequences

\begin{tabular}{ll}
\hline \multicolumn{1}{c}{ peptide } & \multicolumn{1}{c}{ amino acidic sequence } \\
\hline WALP23 & Ac-GWWLALALALALALALALALWWA-NH $\mathrm{N}_{2}$ \\
C(N)-WALP & Ac-CGWWLALALALALALALALALWWA-NH \\
C(C)-WALP & Ac-GWWLALALALALALALALALWWC-NH \\
C12-WALP & Ac-GWWLALALALACALALALALWWA-NH \\
\end{tabular}

linear aggregates in the gel phase of a saturated lipid (DPPC). ${ }^{1,8,9}$ In the liquid-crystalline phase of the same lipid, AFM signatures of aggregation disappear and excimer fluorescence is strongly reduced. ${ }^{1,9}$ Also, in the liquid-crystalline phase of an unsaturated lipid (DOPC) a low level of aggregation was detected by fluorescence. ${ }^{10}$ However, aggregation in the gel phase of DOPC has not been investigated.

Here we present spin-label EPR to probe peptide-membrane interactions in relation to peptide aggregation. We show that it is applicable to different lipid systems over a wide temperature range, including low temperatures where also unsaturated lipids form a gel phase. Spin-label EPR has been used in different ways to investigate membrane-peptide systems, ${ }^{6,11,12}$ but the present approach is novel in that the peptide-peptide interaction is directly probed by spin-labeling the peptide in combination with diamagnetic dilution of the samples.

Diamagnetic dilution is an approach that has previously been used to avoid line broadening by spin-spin interactions. ${ }^{13,14}$ Here we used it for the following reason. In aggregates, the short distances between the spin labels due to the close approach of the peptides causes characteristic changes in the EPR spectra that serve as an observable for aggregation under the membrane condition in question. Because different membrane conditions by themselves also can result in different spectral properties of the spin label, one needs to be able to distinguish these effects from the spin-spin interaction caused by aggregation. In diamagnetically diluted samples, the spin-labeled peptide is diluted by an excess of unlabeled peptide while all other parameters; i.e., the temperature, the type of lipid, and the total lipid-peptide ratio are kept the same. Thus, aggregation can be detected simply as the spectral difference between samples containing labeled peptide only and diamagnetically diluted samples.

Here, the interaction of WALP23 (WALP) with the liquidcrystalline phases and the gel phases of two lipids, DPPC and DOPC, is investigated. The spin label is introduced at a central position of WALP that is within the membrane (SL-WALP) or close to the N- or C-terminal end of WALP (SL-N-WALP or SL-C-WALP). The sequences of these peptides are given in Table 1. Similar to previous studies, ${ }^{1,8,9}$ we find that WALP aggregates in the gel phase of the saturated lipid DPPC. At the measurement temperature of $120 \mathrm{~K}$, cluster aggregates prevail, with no apparent preference for parallel or antiparallel arrangement, whereas other studies, performed at higher temperature, indicated a preference for antiparallel aggregates. ${ }^{1,10,15} \mathrm{We}$ hypothesize that at the lower temperature lipid-lipid interactions are stronger than at $297 \mathrm{~K}$, causing the transition from antiparallel line aggregates to cluster aggregates. We also monitored WALP aggregation for the first time in the gel phase of DOPC and found that aggregation is less extensive than in DPPC. These studies allow us to discriminate between the contributions of specific membrane properties, like the phase of the lipids or the saturation of the lipid fatty acid chains, to peptide aggregation.

\section{Experimental Methods}

Sample Preparation. The lipids DOPC (1,2-dioleoyl-snglycero-3-phosphocholine) and DPPC (1,2-dipalmitoyl-sn-glyc- ero-3-phosphocholine) were purchased from Avanti Polar Lipids (Birmingham, $\mathrm{AL}$ ) and used without further purification.

The spin label reagent, methanethiosulfonate-(1-oxyl-2,2,5,5tetramethylpyrroline- $\Delta 3$-methyl) (MTSL), was purchased from Toronto research chemicals (North York, ON). The reagents used for the synthesis and labeling of the peptides were obtained from commercial sources.

The peptides WALP23, C(N)-WALP, and C(C)-WALP (table 1) were synthesized as described previously. ${ }^{1}$ The peptide $\mathrm{C} 12$ WALP (Table 1) was synthesized manually, using the same synthesis protocol.

The MTSL label was attached to all three cysteine-containing peptides via a disulfide bridge. A typical labeling experiment proceeded as follows: A clear solution of $10 \mu \mathrm{mol}(\sim 25 \mathrm{mg})$ $\mathrm{C}(\mathrm{N})-, \mathrm{C}(\mathrm{C})$-, or C12-WALP in $2.5 \mathrm{~mL}$ of fluoroethanol (TFE) was purged with $\mathrm{N}_{2}$ for $5 \mathrm{~min}$. While the solution was kept under a $\mathrm{N}_{2}$ atmosphere, $10 \mu \mathrm{L}$ of triethylamine and a $\mathrm{N}_{2}$-purged solution of $12.5 \mu \mathrm{mol}(3.3 \mathrm{mg})$ of MTSL in $1 \mathrm{~mL}$ of TFE were added. The reaction mixture was stirred for $48 \mathrm{~h}$ at $4{ }^{\circ} \mathrm{C}$ and in the dark. The labeled peptides, SL-N-WALP, SL-C-WALP and SL-WALP, were precipitated in cold tert-butyl methyl ether/ $n$-hexane (1:1) and lyophilized from tert-butanol/water (1:1) and obtained in yields of at least $72 \%$.

The peptides were characterized by MALDI-ToF (Kratos Axima, ACTH (18-39) external standard, $\alpha$-cyano-4-hydroxycinnamic acid matrix) and electrospray ionization mass spectrometry (ESI-MS, Finnigan LCQ DecaXP Max mass spectrometer operating in positive-ionization mode). The relative intensities of the peaks were not influenced by increased ionization efficiency due to the label, as checked by comparison of mass spectra of (mixtures of) labeled and unlabeled peptides.

In all labeling experiments only the mass of the labeled WALP $\left(\mathrm{Na}^{+}\right.$-ionized form) was found: SL-C-WALP calcd $[\mathrm{M}+\mathrm{H}]_{\text {ave }}^{+} 2793.53$, found $[\mathrm{M}+\mathrm{Na}]_{\text {ave }}^{+}$2815.27. SL-NWALP calcd $[\mathrm{M}+\mathrm{H}]_{\mathrm{ave}}^{+} 2807.55$, found $[\mathrm{M}+\mathrm{Na}]_{\text {ave }}^{+} 2829.19$. SL-WALP calcd $[\mathrm{M}+\mathrm{H}]_{\text {ave }}^{+} 2697.36$, found $[\mathrm{M}+\mathrm{Na}]_{\text {ave }}^{+}$ 2719.48, $[\mathrm{M}+\mathrm{K}]_{\mathrm{ave}}^{+} 2735.46$. On the basis of these results, the labeling-efficiency was estimated to be at least $90 \%$.

Samples were prepared according to ref 11: The lyophilized WALP was dissolved in trifluoroethanol (TFE). The concentration of this stock solution was determined by the absorbance of trp at $280 \mathrm{~nm}$ using an extinction coefficient of $22400 \mathrm{M}^{-1}$ $\mathrm{cm}^{-1}$. The desired amount of WALP stock solution was transferred into a glass vial, and the TFE was evaporated by a nitrogen gas stream. The resulting peptide film was redissolved in $50 \mu \mathrm{L}$ of TFE. This step was repeated one more time. Finally, the peptide film was dissolved in $50 \mu \mathrm{L}$ of TFE and the desired amount of lipids in chloroform was added. The lipid-peptide solution was dried using a nitrogen gas stream followed by vacuum drying overnight. The peptide-lipid film was hydrated in buffer: $50 \mathrm{mM}$ of Tris at $\mathrm{pH} 7.5$ and $100 \mathrm{mM}$ of $\mathrm{NaCl}$. The sample was then subjected to 10 freeze-thaw cycles, using liquid nitrogen and a water bath at $50{ }^{\circ} \mathrm{C}$, to ensure that the lipids alternate between the gel and the liquid-crystalline phase, respectively. The resulting multilamellar vesicles were dispersed by vigorous vortexing before the measurement.

The peptide-lipid ratio (P/L) is given as the molar ratio. Unless otherwise stated the peptide/lipid ratio is $1 / 100$. The concentration of labeled peptides was kept between 100 and $250 \mu \mathrm{M}$ for all measurements, for the measurements at $120 \mathrm{~K}$ $20 \%$ glycerol was added to obtain a frozen glass. The diamagnetically diluted samples (dd-SL-WALP) contain the same amount of SL-WALP, but additional unlabeled WALP to result in a fraction of $10 \%$ or $20 \%$ labeled peptides. 
TABLE 2: Parameters from the Simulation $\left(\tau_{c}, \Delta B\right)$ and the Second Moment Analysis $\left(\left\langle\Delta B_{\mathrm{i}}^{2}\right\rangle,\left\langle\Delta \Delta B^{2}\right\rangle\right)$ of Pure SL-WALP and dd-SL-WALP in DOPC and DPPC

\begin{tabular}{|c|c|c|c|c|c|}
\hline sample & temp $(\mathrm{K})$ & correlation time $\tau_{\mathrm{c}}(\mathrm{ns})^{a}$ & line width $\Delta B(\mathrm{~T})^{b}$ & second moment $\left\langle\Delta B_{\mathrm{i}}{ }^{2}\right\rangle\left(\mathrm{T}^{2}\right)^{c, d}$ & broadening $\left\langle\Delta \triangle B^{2}\right\rangle\left(\mathrm{T}^{2}\right)$ \\
\hline dd-SL-WALP DPPC & 120 & & & $2.8 \times 10^{-6}$ & \\
\hline SL-WALP DPPC & 120 & & & $7.3 \times 10^{-6}$ & $4.5 \times 10^{-6}$ \\
\hline dd-SL-WALP DPPC & 290 & 5.8 & $4.2 \times 10^{-4}$ & $2.6 \times 10^{-6}$ & \\
\hline SL-WALP DPPC & 290 & 5.8 & $8.1 \times 10^{-4}$ & $4.6 \times 10^{-6}$ & $2.0 \times 10^{-6}$ \\
\hline dd-SL-WALP DPPC & 308 & 2.3 & $1.2 \times 10^{-4}$ & $2.1 \times 10^{-6}$ & \\
\hline SL-WALP DPPC & 308 & 2.3 & $2.8 \times 10^{-4}$ & $3.2 \times 10^{-6}$ & $1.1 \times 10^{-6}$ \\
\hline dd-SL-WALP DPPC & 333 & 1.2 & $1.1 \times 10^{-4}$ & $2.3 \times 10^{-6}$ & \\
\hline SL-WALP DPPC & 333 & 1.2 & $1.3 \times 10^{-4}$ & $2.4 \times 10^{-6}$ & $0.1 \times 10^{-6}$ \\
\hline dd-SL-WALP DOPC & 120 & & & $2.8 \times 10^{-6}$ & \\
\hline SL-WALP DOPC & 120 & & & $5.4 \times 10^{-6}$ & $2.6 \times 10^{-6}$ \\
\hline dd-SL-WALP DOPC & 240 & & & $2.5 \times 10^{-6}$ & \\
\hline SL-WALP DOPC & 240 & & & $3.3 \times 10^{-6}$ & $0.8 \times 10^{-6}$ \\
\hline dd-SL-WALP DOPC & 260 & & & $2.1 \times 10^{-6}$ & \\
\hline SL-WALP DOPC & 260 & & & $2.0 \times 10^{-6}$ & $-0.1 \times 10^{-6}$ \\
\hline dd-SL-WALP DOPC & 290 & 2.2 & $1.1 \times 10^{-4}$ & $2.2 \times 10^{-6}$ & \\
\hline SL-WALP DOPC & 290 & 2.2 & $1.8 \times 10^{-4}$ & $2.4 \times 10^{-6}$ & $0.2 \times 10^{-6}$ \\
\hline
\end{tabular}

${ }^{a}$ The rotation correlation time $\left(\tau_{\mathrm{c}}\right)$ was not determined for immobilized spectra $(120 \mathrm{~K})$, the error in $\tau_{\mathrm{c}}$ is $\pm 0.1 \mathrm{~ns} .{ }^{b}$ The intrinsic linewidth $(\triangle B)$ was not determined for immobilized spectra $(120 \mathrm{~K})$, the error in $\Delta B$ is $\pm 0.1 \times 10^{-4} \mathrm{~T}$. ${ }^{c}$ The subscript $\mathrm{i}$ indicates the second moment for the spectra with and without spin-spin interaction. ${ }^{d}$ The second moment $\left\langle\Delta B_{\mathrm{i}}{ }^{2}\right\rangle$ error is $\pm 0.2 \times 10^{-6} \mathrm{~T}^{2}$.

TABLE 3: Parameters from Second Moment Analysis $\left(\left\langle\Delta B_{\mathrm{i}}{ }^{2}\right\rangle,\left\langle\Delta \Delta B^{2}\right\rangle\right)$ of Pure SL-C-WALP and SL-C/N-WALP and dd-SL-C-WALP and dd-SL-C/N-WALP in DPPC

\begin{tabular}{lccc}
\hline \multicolumn{1}{c}{ sample } & temp $(\mathrm{K})$ & $\begin{array}{c}\text { second moment } \\
\left\langle\triangle B_{\mathrm{i}}{ }^{2}\right\rangle\left(\mathrm{T}^{2}\right)^{a, b}\end{array}$ & $\begin{array}{c}\text { broadening } \\
\left\langle\Delta \triangle B^{2}\right\rangle\left(\mathrm{T}^{2}\right)\end{array}$ \\
\hline dd-SL-C-WALP DPPC & 120 & $4.0 \times 10^{-6}$ & \\
SL-C-WALP DPPC & 120 & $5.1 \times 10^{-6}$ & $1.1 \times 10^{-6}$ \\
dd-SL-N/C-WALP DPPC & 120 & $4.0 \times 10^{-6}$ & \\
SL-N/C-WALP DPPC & 120 & $5.1 \times 10^{-6}$. & $1.1 \times 10^{-6}$
\end{tabular}

${ }^{a}$ The subscript $\mathrm{i}$ indicates the second moment for the spectra with and without spin-spin interaction. ${ }^{b}$ The second moment $\left\langle\Delta B_{\mathrm{i}}{ }^{2}\right\rangle$ error is $\pm 0.2 \times 10^{-6} \mathrm{~T}^{2}$.

EPR Experiments. The X-band continuous wave (cw) EPR measurements were performed using an ELEXSYS E 680 spectrometer (Bruker, Rheinstetten, GE) equipped with a helium gas-flow cryostat and a rectangular cavity. For the measurements in frozen solution, $4 \mathrm{~mm}$ outer diameter quartz sample tubes were used and samples were frozen in liquid nitrogen before inserting them in the precooled helium gas-flow cryostat. These EPR spectra were recorded using a modulation amplitude of 2 $\mathrm{G}$ and a microwave attenuation of at least $23 \mathrm{~dB}$. Typical accumulation times have been $28 \mathrm{~min}$.

For measurements in liquid solution, where capillaries of 1.3 $\mathrm{mm}$ inner diameter were used, the modulation amplitude was adjusted to avoid line shape distortions. Typical values are 0.4 G. To control the sample temperature during measurements above room temperature, a nitrogen gas-flow cryostat was used. The EPR spectra were recorded using a microwave (mw) attenuation of $15 \mathrm{~dB}$; the total measurement time was up to 70 min.

All spectra were acquired using a modulation frequency of $100 \mathrm{kHz}$. The spectra were baseline corrected and normalized to the same number of spins (see Tables 2 and 3 for the measurement temperatures)

Simulation of the EPR Spectra. The cw EPR solution spectra were simulated using EasySpin 2.6.0. ${ }^{16}$ For all simulations the following tensor values were used: $\boldsymbol{G}=\left[g_{x x} g_{y y} g_{z z}\right]$ $=\left[\begin{array}{l}2.00906 \\ 2.00687\end{array} 2.00300\right] ;{ }^{17} \boldsymbol{A}=\left[A_{x x} A_{y y} A_{z z}\right]=\left[\begin{array}{lll}13 & 13 & 96\end{array}\right]$ $\mathrm{MHz}$.

A superposition of a mobile component in the fast motion regime and a less mobile component in the slow motion regime was used. The fraction of the mobile component was smaller than $1 \%$.
Second Moment Analysis. Since the line width of the EPR spectra depends among other factors also on the spin-spin interaction, the spectral second moment was used to quantify this interaction. In all experiments, a sample of a monomer, with a spectrum $S_{\mathrm{M}}(B)$ was compared to a sample in which the spin-spin interaction is present $S_{\mathrm{D}}(B)$. The difference of spectral second moments $\left\langle\Delta B^{2}\right\rangle$ was calculated according to the following equation: ${ }^{18}$

$$
\left\langle\Delta \Delta B^{2}\right\rangle=\Delta B_{\mathrm{D}^{2}}-\Delta B_{\mathrm{M}^{2}}=\frac{\int\left(B-B_{\mathrm{FD}}\right)^{2} S_{\mathrm{D}}(B) \mathrm{d} B}{\int S_{\mathrm{D}}(B) \mathrm{d} B}-
$$

where $S_{\mathrm{D}}(B)$ is the absorption spectrum broadened due to dipolar spin-spin interaction, $S_{\mathrm{M}}(B)$ is the absorption spectrum without spin-spin-interaction, $B_{\mathrm{Fi}}$ and $B$ are the first spectral moments and the magnetic field, respectively. Second moments are defined as $\left\langle\triangle B_{\mathrm{M}}{ }^{2}\right\rangle$ for the noninteracting and $\left\langle\triangle B_{\mathrm{D}}{ }^{2}\right\rangle$ for the interacting case.

The absorption EPR spectra were baseline corrected using Xepr software (Bruker Biospin, Rheinstetten, Germany) and the first and second moment were calculated numerically using MatLab (MathWorks, MA).

\section{Results}

The EPR spectra of SL-WALP were measured at different temperatures in the presence of membranes (multilamellar vesicles) composed of DOPC or DPPC lipids. For analysis of the spectral components, the resulting spectra were simulated using the procedures described in Materials and Methods.

Label Efficiency. The labeling degree and basic characteristics of the WALP samples are best judged by roomtemperature EPR. In these spectra, spin-labeled WALP has a characteristic line shape that can be identified in the simulation as a broadened spectrum (Figure 2: $308 \mathrm{~K}$ ) and a rotation correlation time $\tau_{\mathrm{R}}=2.3 \mathrm{~ns}$ (Table 2). A quantitative comparison of the EPR signal intensity to a reference sample with known 


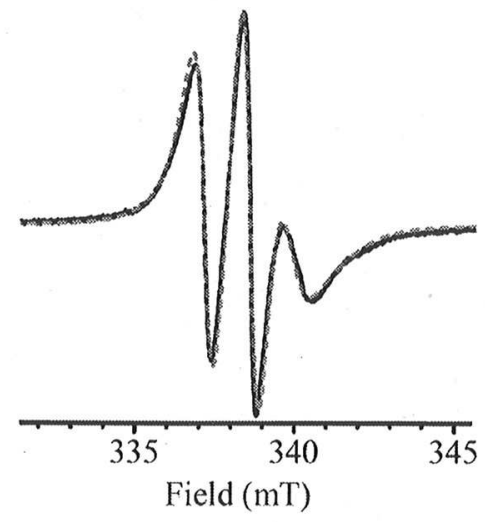

Figure 2. cw EPR spectrum of spin-labeled WALP at $308 \mathrm{~K}$ and its simulation. Pure SL-WALP in DPPC (solid line); simulation (dashed line) (for the simulation parameters, see the Experimental Methods and Table 1).

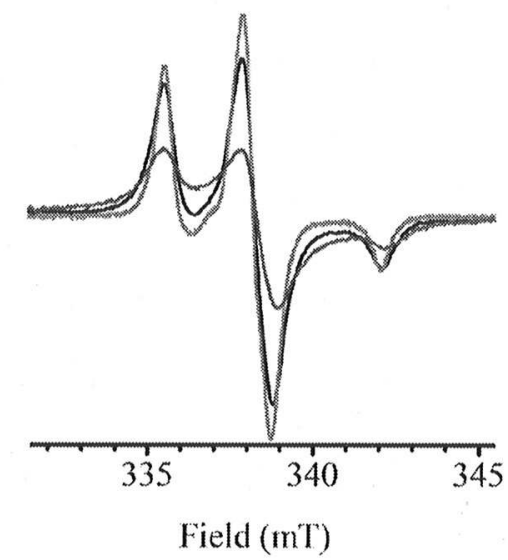

Figure 3. cw EPR spectra of spin-labeled WALP at $120 \mathrm{~K}$. Pure SLWALP in DOPC (black line): microwave (mw) attenuation, $29 \mathrm{~dB}$ total acquisition time, 14 min. Pure SL-WALP in DPPC (blue line). mw attenuation, $23 \mathrm{~dB}$; total measurement time, $28 \mathrm{~min}$. dd-SL-WALP $(10 \%)$ in DPPC (red line): $\mathrm{mw}$ attenuation, $38 \mathrm{~dB}$; total measurement time, 56 min. All spectra, P/L: 1/100. Spectra are normalized to the same number of spins (for the remaining experimental parameters, see the Experimental Methods).

spin concentration revealed that more than $90 \%$ of the peptides are spin labeled, in agreement with the mass-spectrometry results (see Material and Methods).

Additionally, a fast motion component is required in the simulation, which is attributed to a fraction of free MTSL. In all cases, this fraction is below $1 \%$, showing that free MTSL is efficiently removed by the postlabeling purification procedure.

EPR at $120 \mathrm{~K}$. Figure 3 shows EPR frozen solution spectra $(T=120 \mathrm{~K})$ of pure SL-WALP in DOPC and DPPC and of diamagnetically diluted (dd) SL-WALP in DPPC. The spectrum of dd-SL-WALP in DOPC, not shown in the picture, is identical to the one in DPPC. The phase-transition temperature $\left(T_{\mathrm{m}}\right)$ between the liquid-crystalline phase (higher temperature) and the gel phase (lower temperature), of DOPC and DPPC is 253 and $314 \mathrm{~K}$ respectively, ${ }^{19}$ so at the measurement temperature of $120 \mathrm{~K}$ both lipids are in the gel phase. Both dd-SL-WALP and SL-WALP spectra can be well simulated as immobilized powder spectra. From the dd-SL-WALP spectra the hyperfine tensor (A) and the $g$-tensor (G) parameters are obtained. The spectra are normalized to the same number of spins; therefore, the smaller amplitude of the spectra of SL-WALP in DPPC and in DOPC relative to the spectrum of dd-SL-WALP reflects the broadening of the spectra of the SL-WALP samples. For SLWALP in DPPC, the spectra are more broadened than those of the SL-WALP in DOPC. To test the conditions for diamagnetic dilution, a sample composed of $10 \%$ SL-WALP and $90 \%$ WALP (10\% dd-SL-WALP) was compared to a mixture of $20 \%$ SL-WALP with $80 \%$ WALP in DPPC (20\% dd-SL-WALP). The spectra of both samples were identical, showing that spin-spin interactions do not contribute to the line shape for $10 \%$ and $20 \%$ dd-WALP. In the following, both $10 \%$ and $20 \%$ SL-WALP are referred to as dd-SL-WALP.

The width of the spectra is given by the second moment of the spectra $\left(\left\langle\Delta B_{\mathrm{i}}{ }^{2}\right\rangle\right)$, and the broadening is expressed by $\left\langle\Delta B_{\mathrm{D}}{ }^{2}\right\rangle$ $-\left\langle\Delta B_{\mathrm{M}}{ }^{2}\right\rangle=\left\langle\Delta \triangle B^{2}\right\rangle$, where $\left\langle\Delta B_{\mathrm{D}}{ }^{2}\right\rangle$ is the second moment of the SL-WALP sample, $\left\langle\Delta B_{\mathrm{M}}{ }^{2}\right\rangle$ is the second moment of the dd-SL-WALP sample, and $\left\langle\Delta \Delta B^{2}\right\rangle$ the difference in the second moment. The second moments of the EPR spectra are given in Table 2. Positive $\left\langle\triangle \triangle B^{2}\right\rangle$ values will be referred to as broadening. The $\left\langle\triangle \triangle B^{2}\right\rangle$ value of SL-WALP in DPPC is bigger than the value found for the SL-WALP in DOPC. This indicates that at $120 \mathrm{~K} \mathrm{WALP}$ has a higher degree of aggregation in DPPC than in DOPC (see Discussion).

Different P/L ratios of SL-WALP in DOPC and DPPC were measured. For DPPC, the same line shape was observed for $\mathrm{P} / \mathrm{L}$ ratios of $1 / 30$ and $1 / 100$; for DOPC the line was a little wider for the $\mathrm{P} / \mathrm{L}$ ratio of $1 / 30$, without, however, resulting in a significant increase in $\left\langle\Delta \Delta B^{2}\right\rangle$. This indicates that the $\mathrm{P} / \mathrm{L}$ ratios in that range do not significantly affect the spin-spin interaction.

To investigate whether there is a preferred arrangement, i.e., parallel or antiparallel, of the WALP molecules in the membrane, a set of experiments was performed with WALP spin labeled at the N- or the C-terminal positions (SL-N-WALP and SL-C-WALP). The $\left\langle\Delta \triangle B^{2}\right\rangle$ values for SL-N- and SL-C-WALP in DPPC are smaller than those for SL-WALP in the same membrane environment, due to the higher flexibility of the spin label at the end of the WALP-TM helix, yet the broadening remains significant (Table 3). Spectra of SL-N-WALP and SLC-WALP, mixed in the same sample were indistinguishable from the spectra of SL-N-WALP and SL-C-WALP measured separately, suggesting that there is no preferred alignment in DPPC at $120 \mathrm{~K}$ (see Discussion). For DOPC, the SL-N- and SL-C-WALP spectra have such small $\left\langle\Delta \Delta B^{2}\right\rangle$ values that it was not possible to analyze the difference between pure SL-NWALP and SL-C-WALP, respectively, and the mixed sample.

EPR at Temperatures Higher Than $120 \mathrm{~K}$. Below $240 \mathrm{~K}$, both DPPC and DOPC are in the gel phase, and at $120 \mathrm{~K}$, the spectra of dd-SL-WALP revealed complete immobilization. At $240 \mathrm{~K} \mathrm{DOPC}$ is still in the gel phase, but the line shape of dd-SL-WALP (Figure 4a) deviates from that of the immobilized spin label toward a more mobile form. The line-narrowing expected for the higher mobility is also reflected in the trend toward smaller $\left\langle\Delta B^{2}\right\rangle$ values (Table 2 ). Also, the $\left\langle\Delta \Delta B^{2}\right\rangle$ value is reduced relative to the value at $120 \mathrm{~K}$ (for interpretation, see Discussion). At $260 \mathrm{~K}$, above the $T_{\mathrm{m}}$ of DOPC, where the lipids are in the liquid-crystalline phase, the spectra of dd-SL-WALP and SL-WALP are indistinguishable (Figure 4b). The second moment $\left\langle\triangle B^{2}\right\rangle$ of dd-SL-WALP is again reduced relative to the value of $240 \mathrm{~K}$, and it is identical within experimental error to the value of SL-WALP; i.e., $\left\langle\Delta \Delta B^{2}\right\rangle$ vanishes (Table 2). Also, at room temperature $(290 \mathrm{~K})$, no significant broadening was observed (Table 2).

The $T_{\mathrm{m}}$ of DPPC is higher than that of DOPC; therefore, the respective experiments for DPPC are performed at a higher temperature. Parts a and $b$ of Figure 5 show EPR of pure and dd-SL-WALP in DPPC, at $290 \mathrm{~K}$ and at $308 \mathrm{~K}$, i.e., below $T_{\mathrm{m}}$. 
a)

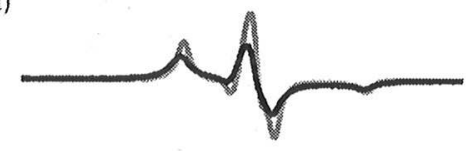

b)

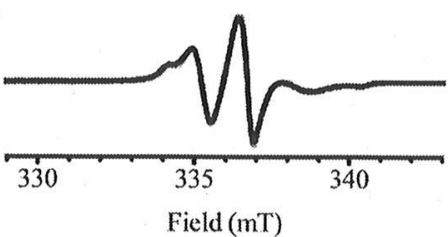

Figure 4. cw EPR spectra of spin-labeled WALP in DOPC, below and above $T_{\mathrm{M}}$. (a) Pure SL-WALP spectrum at $240 \mathrm{~K}$ (black), dd-SLWALP (20\%) spectrum at $240 \mathrm{~K}$ (gray). (b) SL-WALP spectrum at $260 \mathrm{~K}$ (black). dd-SL-WALP (20\%) spectrum at $260 \mathrm{~K}$ (gray). For all spectra, P/L ratio: 1/100. Spectra are normalized to the same number of spins (for the remaining experimental parameters, see the Experimental Methods).

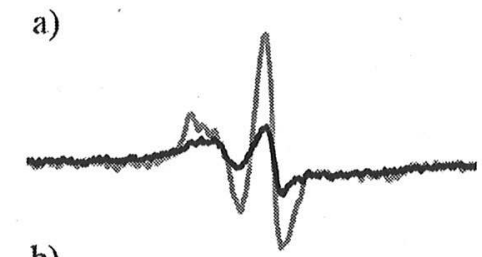

b)

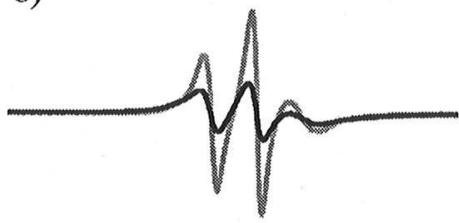

c)
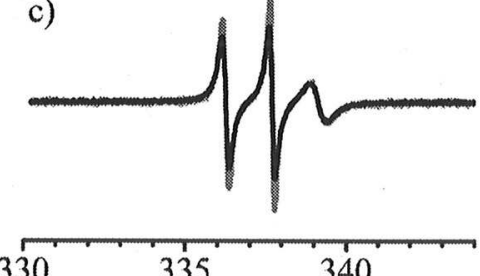

330

340

Field $(\mathrm{mT})$

Figure 5. cw EPR spectra of spin-labeled WALP in DPPC below and above $T_{\mathrm{M}}$. Pure SL-WALP (black) and dd-SL-WALP (20\%, gray) at (a) $T=290 \mathrm{~K}$, (b) $T=308 \mathrm{~K}$, and (c) $T=333 \mathrm{~K}$. P/L ratio: $1 / 100$ Spectra are normalized to the same number of spins (for the remaining experimental parameters, see the Experimental Methods).

At both temperatures, the SL-WALP is significantly broadened with respect to the diamagnetically diluted one. The spectra were simulated using standard liquid solution parameters, resulting in rotation correlation times $\tau_{\mathrm{R}}$ in the nanosecond time regime. Spectra of SL-WALP and dd-SL-WALP under identical membrane conditions could be simulated with identical values for $\tau_{\mathrm{R}}$ but a larger $\Delta B$ for the pure SL-WALP (Table 2). Figure 5c shows EPR liquid-solution spectra of pure and diamagnetically diluted SL-WALP in DPPC at $333 \mathrm{~K}$. At this temperature, which is above the $T_{\mathrm{m}}, \mathrm{DPPC}$ is in the liquid-crystalline phase. The spectrum of SL-WALP (Figure 5c) has a lower intensity, suggesting that the spectrum is slightly broader than the one of dd-SL-WALP. However, this broadening is not sufficient to lead to a $\left\langle\triangle \Delta B^{2}\right\rangle$ value that is significantly above zero (Table 2 ).

Model for the Spin-Spin Interaction. To interpret the measured $\left\langle\Delta \triangle B^{2}\right\rangle$ values of the samples at $120 \mathrm{~K}$, the dipolar interaction was calculated for model aggregates. For a spin pair, the $\left\langle\Delta \Delta B^{2}\right\rangle$ value is related to the distance as

$$
\left\langle\Delta \Delta B^{2}\right\rangle=p \frac{1}{d^{6}}
$$

where $d$ is the spin-spin distance and $p \approx 1.56 \times 10^{-60} T^{2} m^{6} .^{18}$ Aggregates of more than two spins are modeled taking all pair interactions into account

$$
\left\langle\Delta \Delta B^{2}\right\rangle=\sum_{i}\left\langle\Delta \Delta B_{i}{ }^{2}\right\rangle=p \sum_{i} \frac{1}{d_{i}^{6}}
$$

where the second moments of each two-particle interaction are added. ${ }^{20}$ The membrane is assumed to be two-dimensional, and the dipolar broadening due to line aggregates as well as twodimensional cluster aggregates was calculated. The aggregates are modeled as having a fixed spin-spin distance $R$ between nearest neighbors. To illustrate the procedure, a linear trimer is discussed. In this case, the central spin has two neighbors at a distance $R$ and the spins at the ends of the aggregate have one neighbor at the distance $R$ and one neighbor at the distance $2 R$, resulting in the second moment

$$
\left\langle\Delta \Delta B^{2}\right\rangle=p\left(\frac{1}{3} \frac{2}{R^{6}}+\frac{2}{3}\left(\frac{1}{R^{6}}+\frac{1}{(2 R)^{6}}\right)\right)
$$

Similarly, linear aggregates of any size up to the infinitely long line aggregate $\left[\left\langle\Delta \Delta B^{2}\right\rangle=\left(2 \pi^{6} / 945\right)\left(p / R^{6}\right)\right]$ as well as cluster aggregates can be calculated. The interspin distance $R$ in the aggregates is estimated from a model in which the transmembrane helix of one WALP touches the surface of the adjacent WALP. Using a helix diameter of $1 \mathrm{~nm},{ }^{8}$ a distance between spin labels of $R=1 \mathrm{~nm}$ results, if the linker joining the spin label to the WALP-peptide backbone has the same orientation in both peptides. Since the linker length of MTSL in the extended conformation is $0.5 \mathrm{~nm}$ and there is no information on the rotational orientation of the WALP-helices in the membrane, we also investigated the effect of $R$ on the broadening.

As a reference, also the second moment of nonaggregated, i.e., randomly distributed spins in the membrane is needed. The number of spins $N(r) \mathrm{d} r$ at a distance between $r$ and $r+\mathrm{d} r$ from a spin is

$$
N(r)=c 2 \pi r \mathrm{~d} r
$$

with $c$, the density of spins in the membrane. Introducing $R_{0}$, the distance of closest approach, a broadening of

$$
\left\langle\Delta \Delta B^{2}\right\rangle=p \int_{R_{0}}^{\infty} N(r) \frac{1}{r^{6}} \mathrm{~d} r=\frac{1}{2} \pi c p \frac{1}{R_{0}^{4}}
$$

results. The distance of the closest approach corresponds to the helix diameter, i.e., $R=R_{0}$. To obtain an estimate of the magnitude of this broadening, the density $c$ is calculated from the area of the headgroup of the lipids of 82 and $52.3 \AA^{2}$ for DOPC and DPPC, respectively. ${ }^{21,22}$ For a peptide/lipid ratio of $1 / 100$, corresponding to an area of 50 lipid heads in the bilayer per spin, a surface density of $c=\left(41 \mathrm{~nm}^{2}\right)^{-1}$ for DOPC and 


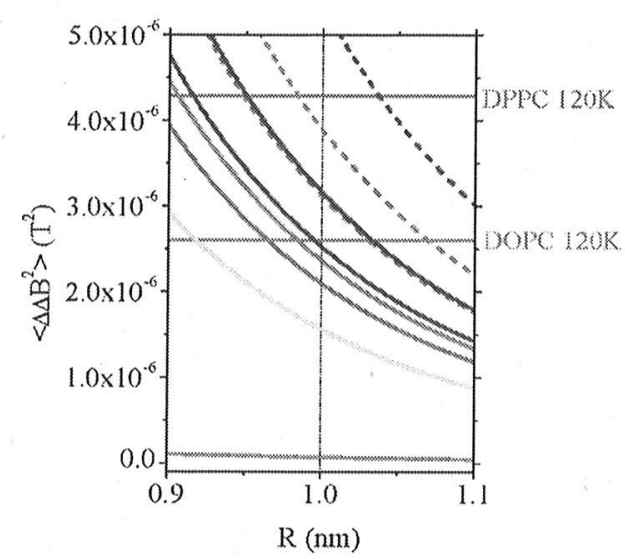

Figure 6. Second moment (spectral broadening owing to dipolar interaction) as a function of the nearest neighbor spin distance $R$ for different model geometries derived from calculations. Linear aggregates are shown as solid lines, in order of increasing broadening containing 2 (dimer, yellow), 3 (trimer, red), 4 (tetramer, green), 5 (pentamer, blue), or an infinite number of spins (purple). Dashed lines correspond to 2 D clusters containing (again in order of increasing broadening) 3 (red), 4 (green), or 7 (blue) molecules, respectively. The broadening for 2D randomly distributed spins as a function of closest approach $\left(R_{0}\right)$ (see text) is visualized by the gray solid line. The experimentally obtained broadening values for spin labeled WALP in model membranes $(\mathrm{P} / \mathrm{L}=1 / 100)$ at different temperatures (see Table 2) are depicted as horizontal lines: DPPC (olive) at $120 \mathrm{~K}$, and DOPC (red) at $120 \mathrm{~K}$. For the cases of non-negligible mobility (horizontal, dashed lines) the broadening may be reduced due to motional averaging (see text).

$\left(26 \mathrm{~nm}^{2}\right)^{-1}$ for DPPC results. Only a rough estimate of this factor will be needed in the following discussion.

In Figure 6 , the results of the calculated $\left\langle\Delta \Delta B^{2}\right\rangle$ values are plotted as a function of $R$. Broadening obtained from model calculations of linear clusters containing 2, 3, 4, and 5, or an infinite number of spin-labeled peptides as well as clusters of 3,4 , and 7 peptides is shown. To calculate the broadening due to a random distribution for Figure 6 , an averaged surface density of $\left(35 \mathrm{~nm}^{2}\right)^{-1}$ was used. Linear aggregates yield smaller degrees of broadening than cluster aggregates, and, due to the $r^{-6}$ dependence of the broadening, short distances dominate, such that, for example, the infinitely long line aggregate has a broadening that is close to the trimeric cluster aggregate.

In Figure 6, also experimental $\left\langle\Lambda \wedge B^{2}\right\rangle$ parameters are shown. Note that a direct comparison is only straightforward for the measurements at $120 \mathrm{~K}$. For higher temperatures (partial) averaging can reduce the broadening, as discussed below. The slight deviation of the absolute values due to incomplete labeling has been neglected.

In the following, we will analyze the experimental results and compare them to the calculation to construct a picture of WALP interaction in DOPC and DPPC.

\section{Discussion}

The goal of this study was to use EPR to investigate whether certain membrane conditions promote peptide-peptide aggregation. In the following we interpret the EPR spectra and develop a model for the arrangement of the peptides in the membrane.

In the gel phase of the lipids, at $120 \mathrm{~K}$, the spin labels are immobilized, and the close approach of the spin labels in the pure SL-WALP causes line broadening due to the dipole-dipole interaction. The broadening is more pronounced for SL-WALP in DPPC than in DOPC, showing that the dipolar interaction is stronger for SL-WALP in DPPC than in DOPC. Model calculations (see Results) relate this broadening, expressed as the difference in second moments $\left\langle\Delta \Delta B^{2}\right\rangle$, to the interaction of spin labels in different types of aggregates, namely cluster aggregates (Figure 1b) and line aggregates (Figure 1c). Given the disordered state of the membrane, such models can only be approximate, but we show that they can be used to classify the states of aggregates in a semiquantitative way. Figure 6 shows the results of the calculations together with the experimental values (olive and red horizontal lines). The broadening of the EPR spectra of SL-WALP in DOPC at $120 \mathrm{~K}$ is comparable to that of a linear aggregate containing five molecules for $R \geq 1$ $\mathrm{nm}$. For SL-WALP in DPPC, $\left\langle\triangle \triangle B^{2}\right\rangle$ is larger and at $R \geq 0.95$ $\mathrm{nm}$ it is in the cluster region of the diagram, close to the tetrameric cluster aggregate. This suggests that, in the gel phase of DPPC, WALP aggregates are likely of the cluster type, whereas in DOPC the aggregates are more likely to be linetype aggregates.

Line-type and cluster aggregates should also have distinct characteristics when $\mathrm{C}$-and $\mathrm{N}$-terminally labeled peptides are compared. For line aggregates with a strictly antiparallel (parallel) arrangement, the mixed sample should have a smaller (larger) interspin distance than the individual SL-C or SL-NWALP samples, resulting in a larger (smaller) $\left\langle\Delta \Delta B^{2}\right\rangle$. For cluster aggregates only very specific configurations can be envisaged that would have a strictly antiparallel arrangement of the peptides, making random orientations more likely.

For WALP in DPPC, no difference in broadening between SL-N and SL-C-WALP measured individually or combined in one sample was observed (see Results). This argues either for a line aggregate with random arrangements of the $\mathrm{C}$ - and $\mathrm{N}$-termini of WALP, i.e., neither strictly parallel nor antiparallel, or for a cluster type aggregate. The latter form would be favored also from the amount of broadening. In DOPC, the broadening of the N- and C-terminal labeled WALP was too small to be detected. For the pure SL-C- or N-WALP samples we attribute this to the long distance between the spin labels in our constructs in the antiparallel orientation. For the SL-C- and N-WALP mixed samples, the long distance and the overall smaller broadening in the line aggregates, compared to the cluster aggregates, makes the broadening too small to be detected. Taking all into account, the simplest interpretation of the data so far would be cluster aggregates for WALP in the gel phase of DPPC and line aggregates for WALP in the gel phase of DOPC.

Before doing the same analysis for the liquid-crystalline phase of the lipids, first the effect of higher temperatures on the spectra has to be discussed. Above $120 \mathrm{~K}$ and particularly at $240 \mathrm{~K}$ and above, the mobility of SL-WALP in the membrane increases, and this could (partially) average the dipolar interaction. Such an averaging can only be excluded, if the correlation time of the interspin vector is long enough to fulfill the following condition: ${ }^{20}$

$$
\tau_{\mathrm{c}}\left(\left\langle\Delta \omega_{0}{ }^{2}\right\rangle\right)^{1 / 2}=\tau_{\mathrm{c}}\left(4 \pi^{2}\left(2.8 \times 10^{10} \frac{H z}{T}\right)^{2}\left\langle\Delta \Delta B_{0}{ }^{2}\right\rangle\right)^{1 / 2} \gg 1
$$

With the broadening $\left\langle\Delta \Delta B^{2}\right\rangle$ obtained from the frozen solution data (Table 2 and 3 ) this condition (eq 7) can be rewritten as

$$
\tau_{\mathrm{c}} \gg \tau_{\mathrm{c}}^{*}
$$

with the critical correlation time $\tau_{c}^{*}$. Assuming that the correlation time of the interspin vector corresponds to the spin-label 
rotational correlation time, for temperatures above $240 \mathrm{~K}$ one obtains the corresponding ratio to be in the range $\tau_{\mathrm{c}} / \tau_{\mathrm{c}}^{*} \approx$ $0.4-1.7$, i.e., far from the requirement stated in eq 8 . Therefore, the broadening could be reduced by motional narrowing.

For WALP at 290 and $308 \mathrm{~K}$ in DPPC, the membrane is still in the gel phase but the spectra of dd-SL-WALP are narrowed relative to those at $120 \mathrm{~K}$, revealing the increased mobility of the spin label under these conditions. Also, $\left\langle\Delta \Delta B^{2}\right\rangle$ decreases, but it remains significantly larger than the $\left\langle\triangle \Delta B^{2}\right\rangle$ expected for a random distribution of isolated spins in the membrane, a clear indication of aggregation. The reduction in $\left\langle\Delta \triangle B^{2}\right\rangle$ could be caused by (partial) averaging of the dipolar interaction and/or a conversion of cluster aggregates into smaller aggregates or line-type aggregates. The difficulty to quantify the contribution of both factors to the reduction in $\left\langle\triangle \triangle B^{2}\right\rangle$ makes it impossible to discriminate between both effects and to determine a possible change in aggregate size or shape.

In the liquid-crystalline state $(T=333 \mathrm{~K}),\left\langle\Delta \Delta B^{2}\right\rangle$ is much smaller than at $308 \mathrm{~K}$, while the experimentally determined $\tau_{\mathrm{c}}$ values are only slightly smaller than those at $308 \mathrm{~K}$, where the membrane is in the gel state. The decreased broadening at temperatures above the phase transition is still significant even if one would assume a linear decrease of $\left(\left\langle\Delta \Delta B^{2}\right\rangle\right)^{1 / 2}$ with $\tau_{\mathrm{c}}$. Hence, it is plausible that the dipolar interaction not only is averaged but also is indeed smaller, and that would suggest less or no aggregation in the liquid-crystalline state of the membrane. This hypothesis is supported by the fact that the broadening increases proportionally to the peptide lipid ratio in a range between $1 / 100$ and 1/30 (data not shown), as expected for randomly distributed spins (eq 6). For SL-WALP in DOPC $\left\langle\triangle \triangle B^{2}\right\rangle$ follows a similar pattern as in DPPC, suggesting that also in DOPC there is aggregation below, but not above $T_{\mathrm{M}}$. However, in DPPC the tendency to aggregate below $T_{\mathrm{M}}$ seems larger than for DOPC.

If aggregation occurs only in the gel phase, when the samples are cooled through $T_{\mathrm{M}}$ to create the gel phase, the peptides, which are randomly distributed in the membrane in the liquidcrystalline phase, must diffuse toward each other when the membrane enters the gel phase. This requires that the peptide diffusion in the membrane is faster than cooling through the phase transition, a condition obviously met in the cooling protocol used in the present study.

In summary, we conclude that, at $120 \mathrm{~K}$, WALP aggregates in the gel phase of DPPC most likely as clusters and in DOPC as line aggregates, but not in the liquid crystalline phase of the lipids.

Previously, aggregation of WALP in the gel phase of DPPC had been observed by atomic force microscopy (AFM). Linetype depressions were observed by $\mathrm{AFM}^{1,8,10}$ and interpreted as linear aggregates of WALP. Striated domains are made up of parallel line depressions in which the lines are separated by $8 \mathrm{~nm}$. These results suggest that the EPR parameters should only reveal linear aggregates, as the distance to an adjacent line in striated domains is too large to cause broadening according to the model used for the two-dimensional aggregates. The line aggregate model would allow for close contact of maximally four WALP peptides at the bifurcation points of line depressions, but such points should only represent a minority of the sites. ${ }^{1}$ Fluorescence studies of the same system revealed antiparallel aggregates that, in the light of the AFM results, may also be interpreted as line aggregates. ${ }^{1,10}$

An obvious difference between AFM and fluorescence experiments on the one hand and the EPR experiments on the other hand is that the latter experiments are performed at significantly lower temperatures $(120 \mathrm{~K}$ for EPR, AFM and fluorescence not below $297 \mathrm{~K}$ ). If, when the temperature is lowered, lipid-lipid interactions become stronger, the lipids could force the peptides into cluster type aggregates, as that would decrease the lipid-peptide interaction surface. This would require the peptides to give up the more favorable antiparallel arrangement possible only in the line aggregates. The smaller $\left\langle\Delta \Delta B^{2}\right\rangle$ values at higher temperatures are consistent with a reduction in cluster size and conversion to line aggregates, but the increased mobility of the peptides at higher temperatures makes it impossible to determine that factor.

The AFM results also showed that aggregate signatures disappear upon heating the DPPC sample above the phase transition into the liquid crystalline phase. ${ }^{1}$ Similarly, the yield of excimers decreases sharply upon heating above $T_{\mathrm{M}}$, showing that aggregates that were present in the gel phase, i.e., below $T_{\mathrm{M}}$, break up in the liquid-crystalline phase. ${ }^{1}$ The remaining excimer contribution at higher temperatures, on the order of $5 \%$, is likely to be too small to be detected by EPR, because in the liquid-crystalline phase partial averaging of the dipolar interaction interferes. To estimate the minimum amount of aggregated SL-WALP that could be detected in the liquidcrystalline phase under these conditions is difficult, but a minimum of $30 \%$ seems likely. This would make it impossible to detect the amount of aggregation found in the fluorescence studies.

Our study provides the first experimental data about aggregation of WALP in the gel phase of DOPC, and our data suggest linear aggregates at $120 \mathrm{~K}$. In the liquid-crystalline phase, excimer formation of WALP, suggesting dimers or higher aggregates, had been found for $\mathrm{P} / \mathrm{L}$ ratios above $1 / 50,{ }^{10}$ but also smaller amounts of aggregates were observed for $\mathrm{P} / \mathrm{L}$ ratios $1 / 100 .^{1}$ But again, the yield of excimers found by optical methods was small (around 5\%) ${ }^{1}$ and thus below the detection limit of EPR in the liquid crystalline phase of the membrane.

The emerging picture of aggregation of WALP in the two membranes is the following: Previously, aggregation of WALP in the gel phase of DPPC was found by AFM, optical spectroscopy, and NMR. ${ }^{1,8}$ Our observations of SL-WALP at $120 \mathrm{~K}$ agree with these results, although our interpretation suggests cluster rather than line aggregates. We propose that at the temperature of the EPR experiments cluster aggregates prevail, because lipid-lipid interactions become stronger at lower temperatures. Even though the peptide-peptide interaction seems to favor an antiparallel arrangement of the peptides, ${ }^{10,15}$ this energy gain could be offset by the increasingly unfavorable lipid-peptide interaction. Under these circumstances, cluster aggregates that minimize the lipid-peptide interface could be the optimal solution.

Aggregation in the gel phase of DOPC had not been reported before. The aggregation of the peptides in DPPC was attributed to the balance of lipid-lipid and lipid-peptide interactions in the gel phase of the saturated lipid DPPC, particularly, by the increased ordering of the lipid chains in the vicinity of WALP found by NMR. ${ }^{1}$ Aggregation in the gel phase of DOPC indicates that the lipid order in the gel phase is sufficient to induce aggregation of WALP, even though the lipids in the gel phase of DOPC, an unsaturated lipid, should be less well ordered than those of DPPC, the saturated one. In other words, the results presented here suggest that the gel phase is more important than the precise structure of the lipid in the vicinity of WALP.

\section{Conclusions}

The approach to combine EPR line broadening, diamagnetic dilution and model calculation provides information on ag- 
gregate shape and, in certain cases, also the size of the aggregates. This approach should be applicable to a wide range of membrane conditions.

The higher degree of order of the lipid chains in the gel phase seems to be a major factor in promoting aggregation because both the saturated lipid DPPC and the unsaturated lipid DOPC promote WALP aggregation in that phase. However, lipid saturation seems to affect the type of aggregation and our analysis suggests cluster aggregates in DPPC and line aggregates in DOPC. The strength of lipid-peptide interactions may also depend on temperature. In DPPC, EPR at $120 \mathrm{~K}$ indicates cluster aggregates, whereas AFM at $297 \mathrm{~K}$ reveals line aggregates.

The degree of aggregation must be significantly smaller in the liquid-crystalline phase of both lipids, but experimental limitations in the fluid, mobile regime of the membrane limit the sensitivity and uniqueness of the answers obtained. Such limitations can, in principle, be overcome by a quantitative analysis of the peptide mobility in the membrane.

The EPR approach presented is suitable to investigate the state of peptides in membrane. It should be applicable to a wide range of membrane conditions. We demonstrate it for the gel phase of two lipids. In view of the occurrence of gel-phaselike states in biological membranes, this presents a relevant step ahead in the study of lipid-peptide interaction.

Acknowledgment. We thank Emma Sparr and Thomas Nyholm for their contributions to earlier stages of the project. Edgar Groenen is acknowledged for constant interest, support and fruitful discussions. Support by the Dutch Science Organization (NWO-Top grants 700.54.303 (T.R.-M.) and 700.53.305 (F.S., M.H.)), a Marie Curie Early Stage Trainning Fellowship from the European Community's Sixth Framework program (Biomem-MEST-CT 2004-007931 (A.H.)), and a fellowship by the Deutsche Forschungsgemeinschaft (Dr 743/1-1 (M.D.)) are gratefully acknowledged. This work is part of the research program of the Stichting voor fundamenteel onderzoek van de materie (FOM, program Biomolecular Physics 03BM03 (MH)), which is financially supported by NWO.

\section{References and Notes}

(1) Sparr, E.; Ganchev, D. N.; Snel, M. M. E.; Ridder, A. N. J. A.; Kroon-Batenburg, L. M. J.; Chupin, V.; Rijkers, D. T. S.; Killian, J. A.; de Kruijff, B. Molecular organization in striated domains induced by transmembrane alpha-helical peptides in dipalmitoyl phosphatidylcholine bilayers. Biochemistry 2005, 44 (1), 2-10.

(2) Killian, J. A.; Nyholm, T. K. M. Peptides in lipid bilayers: the power of simple models. Curr. Opin. Struct. Biol. 2006, 16 (4), 473-479.

(3) Ozdirekcan, S.; Etchebest, C.; Killian, J. A.; Fuchs, P. F. J. On the orientation of a designed transmembrane peptide: Toward the right tilt angle. J. Am. Chem. Soc. 2007, 129 (49), 15174-15181.
(4) Lemaitre, V.; de Planque, M. R. R.; Howes, A. P.; Smith, M. E.; Dupree, R ; Watts, A. Solid-state O-17 NMR as a probe for structural studies of proteins in biomembranes. J. Am. Chem. Soc. 2004, 126 (47), 1532015321

(5) Holt, A.; de Almeida, R. F. M.; Nyholm, T. K. M.; Loura, L. M. S.; Daily, A. E.; Staffhorst, R. W. H. M.; Rijkers, D. T. S.; Koeppe, R. E.; Prieto, M.; Killian, J. A. Is there a preferential interaction between cholesterol and tryptophan residues in membrane proteins. Biochemistry 2008, 47 (8), 2638-2649.

(6) Nielsen, R. D.; Che, K. P.; Gelb, M. H.; Robinson, B. H. A ruler for determining the position of proteins in membranes. J. Am. Chem. Soc. 2005, 127 (17), 6430-6442.

(7) Killian, J. A.; Salemink, I.; dePlanque, M. R. R.; Lindblom, G.; Koeppe, R. E.; Greathouse, D. V. Induction of nonbilayer structures in diacylphosphatidylcholine model membranes by transmembrane alphahelical peptides: Importance of hydrophobic mismatch and proposed role of tryptophans. Biochemistry 1996, 35 (3), 1037-1045

(8) Rinia, H. A.; Kik, R. A.; Demel, R. A.; Snel, M. M. E; Killian, J. A.; van der Eerden, J. P. J. M.; de Kruijff, B. Visualization of highly ordered striated domains induced by transmembrane peptides in supported phosphatidylcholine bilayers. Biochemistry 2000, 39 (19), 5852-5858.

(9) de Kruijff, B.; Killian, J. A.; Ganchev, D. N.; Rinia, H. A.; Sparr, E. Striated domains: self-organizing ordered assemblies of transmembrane alpha-helical peptides and lipids in bilayers. Biol. Chem. 2006, 387 (3), 235-241.

(10) Sparr, E.; Ash, W. L.; Nazarov, P. V.; Rijkers, D. T. S.; Hemminga, M. A.; Tieleman, D. P.; Killian, J. A. Self-association of transmembrane alpha-helices in model membranes - Importance of helix orientation and role of hydrophobic mismatch. J. Biol. Chem. 2005, 280 (47), 39324-39331.

(11) de Planque, M. R. R.; Greathouse, D. V.; Koeppe, R. E.; Schäfer, H.; Marsh, D.; Killian, J. A. Influence of lipid/peptide hydrophobic mismatch on the thickness of diacylphosphatidylcholine bilayers. A H-2 NMR and ESR study using designed transmembrane alpha-helical peptides and gramicidin A. Biochemistry 1998, 37 (26), 9333-9345.

(12) Bashtovyy, D.; Marsh, D.; Hemminga, M. A.; Pali, T. Constrained modeling of spin-labeled major coat protein mutants from M13 bacteriophage in a phospholipid bilayer. Protein Sci. 2001, 10 (5), 979-987.

(13) Margittai, M.; Langen, R. Spin labeling analysis of amyloids and other protein aggregates. Amyloid, Prions, Other Protein Aggregates, Pt. C 2006, 413, 122-139.

(14) Der-Sarkissian, A.; Jao, C. C.; Chen, J.; Langen, R. Structural organization of alpha-synuclein fibrils studied by site-directed spin labeling. J. Biol. Chem. 2003, 278 (39), 37530-37535.

(15) Yano, Y.; Matsuzaki, K. Measurement of thermodynamic parameters for hydrophobic mismatch 1: Self-association of a transmembrane helix. Biochemistry 2006, 45 (10), 3370-3378.

(16) Stoll, S.; Schweiger, A. EasySpin, a comprehensive software package for spectral simulation and analysis in EPR. J. Magn. Reson. 2006 , 178 (1), 42-55

(17) Steigmiller, S.; Börsch, M.; Gräber, P.; Huber, M. Distances between the b-subunits in the tether domain of F0F1-ATP synthase from E. coli. Biochim. Biophys. Acta-Bioenerg. 2005, 1708 (2), 143-153.

(18) Steinhoff, H. J. Methods for study of protein dynamics and proteinprotein interaction in protein-ubiquitination by electron paramagnetic resonance spectroscopy. Frontiers Biosci. 2002, 7, C97-C110.

(19) Lipid-Protein Interactions; John Wiley \& Sons: New York, 1982.

(20) Abragam, A. The Principles of Nuclear Magnetism; Oxford University Press: Oxford, U.K., 1961.

(21) Nägle, J. F.; Tristram-Nägle, S. Structure of lipid bilayers. Biochim. Biophys. Acta-Rev. Biomembr. 2000, 1469 (3), 159-195.

(22) Lis, L. J.; Mcalister, M.; Fuller, N.; Rand, R. P.; Parsegian, V. A. Interactions Between Neutral Phospholipid-Bilayer Membranes. Biophys J. 1982, 37 (3), 657-665. 